\title{
BMJ Open German-wide prospective DACAPO cohort of survivors of the acute respiratory distress syndrome (ARDS): a cohort profile
}

\begin{abstract}
Frank Dodoo-Schittko, ${ }^{1}$ Susanne Brandstetter, ${ }^{1}$ Magdalena Brandl, ${ }^{1}$ Sebastian Blecha, ${ }^{2}$ Michael Quintel, ${ }^{3}$ Steffen Weber-Carstens, ${ }^{4}$ Stefan Kluge, ${ }^{5}$ Thomas Kirschning, ${ }^{6}$ Thomas Muders, ${ }^{7}$ Sven Bercker, ${ }^{8}$ Björn Ellger, ${ }^{9}$ Christian Arndt, ${ }^{10}$ Patrick Meybohm, ${ }^{11}$ Michael Adamzik, ${ }^{12}$ Anton Goldmann, ${ }^{4}$ Christian Karagiannidis, ${ }^{13}$ Thomas Bein, ${ }^{2}$ Christian Apfelbacher, ${ }^{1}$ the DACAPO Study Group
\end{abstract}

To cite: Dodoo-Schittko F, Brandstetter S, Brandl M, et al. German-wide prospective DACAPO cohort of survivors of the acute respiratory distress syndrome (ARDS): a cohort profile. BMJ Open 2018;8:e019342. doi:10.1136/ bmjopen-2017-019342

- Prepublication history for this paper is available online. To view these files, please visit the journal online (http://dx.doi org/10.1136/bmjopen-2017019342).

Received 3 September 2017 Revised 8 January 2018 Accepted 31 January 2018

Check for updates

For numbered affiliations see end of article.

Correspondence to Dr Frank Dodoo-Schittko; frank.dodoo-schittko@klinik. uni-regensburg.de

\section{ABSTRACT}

Purpose While most research focuses on the association between medical characteristics and residual morbidity of survivors of the acute respiratory distress syndrome (ARDS), little is known about the relation between potentially modifiable intensive care unit (ICU) features and the course of health-related quality of life (HRQOL). Accordingly, the DACAPO study was set up to elucidate the influence of quality of intensive care on HRQOL and return to work (RtW) in survivors of ARDS. The continued follow-up of these former ICU patients leads to the establishment of the DACAPO (survivor) cohort.

Participants Sixty-one ICUs all over Germany recruited patients with ARDS between September 2014 and April 2016. Inclusion criteria were: (1) age older than 18 years and (2) ARDS diagnosis according to the 'Berlin definition'. No further inclusion or exclusion criteria were applied. 1225 patients with ARDS could be included in the DACAPO ICU sample. Subsequently, the 876 survivors at ICU discharge form the actual DACAPO cohort.

Findings to date The recruitment of the participants of the DACAPO cohort and the baseline data collection has been completed. The care-related data of the DACAPO cohort reveal a high proportion of adverse events (in particular, hypoglycaemia and reintubation). However, evidence-based supportive measures were applied frequently.

Future plans Three months, 6 months and 1 year after ICU admission a follow-up assessment is conducted. The instruments of the follow-up questionnaires comprise the domains: (A) HRQoL, (B) RtW, (C) general disability, (D) psychiatric symptoms and (E) social support. Additionally, an annual follow-up of the DACAPO cohort focusing on HRQoL, psychiatric symptoms and healthcare utilisation will be conducted. Furthermore, several add-on projects affecting medical issues are envisaged.

Trial registration number NCT02637011.
Strengths and limitations of this study

This is one of the few large acute respiratory distress syndrome (ARDS) survivor cohorts applying the current diagnostic criteria ('Berlin definition').

- This is the first large ARDS survivor cohort with explicit focus on health-related quality of life, characterisation of sociodemographic aspects and healthcare utilisation.

The cohort is characterised by a wide range of disease-related and care-related information during intensive care unit (ICU) phase.

- It cannot be assumed that in the participating ICUs all patients with ARDS had the same probability to be included in the cohort (risk of selection bias).

\section{INTRODUCTION}

Acute respiratory distress syndrome (ARDS) is a serious condition characterised by life-threatening damage of the lung parenchyma. ${ }^{1}$ The management of ARDS requires comprehensive intensive care unit (ICU) treatment including mandatory mechanical ventilation. The diagnostic criteria of ARDS according to the 'Berlin definition' include acute onset and radiological evidence of bilateral infiltrates, which cannot be fully explained by cardiac failure or fluid overload and hypoxaemia. ${ }^{2}$ Based on the degree of hypoxaemia, three categories of ARDS (mild, moderate and severe) are determined by the current 'Berlin definition'. ${ }^{2}$ About $10 \%$ of ICU patients develop ARDS $^{3}$ and despite optimal treatment, including prone positioning $^{4}$ and extracorporeal membrane oxygenation $(\mathrm{ECMO})^{5}$ for the most severe forms, recent hospital mortality estimates are as high as $46 \%$. $^{3}$ Above and beyond mortality, 
many ARDS survivors suffer from long-term persistent physical and mental morbidity. ${ }^{6}$ A systematic review with subsequent meta-analysis ${ }^{6}$ revealed lower pooled estimated scores for all health-related quality of life (HRQoL) domains of the Medical Outcomes Study Short Form 36-Item Health Survey in ARDS survivors compared with general population. ${ }^{7}$ Even 5 years after ICU discharge, ARDS survivors have decreased HRQoL and a reduced 6 min walk distance compared with mean norm scores. ${ }^{8}$ Among the mental disorders, increased prevalence rates are reported for depression, post-traumatic stress disorder (PTSD) and anxiety disorder in former patients with ARDS. ${ }^{910}$ Taken together, there is a growing body of evidence for decreased HRQoL and disability, but little is known about determinants of clinical relevance for HRQoL and return to work (RtW) in survivors of ARDS. ${ }^{11}$ Furthermore, none of the large ARDS cohorts provide sufficient information on sociodemographic characteristics of patients with ARDS and ARDS survivors. ${ }^{12}$ Against this background, an ARDS survivor cohort that allows investigation into the influences of hospital quality of care (QoC) and subsequent healthcare utilisation (HCU) on prolonged mental and physical morbidity, HRQoL and RtW is highly desirable.

\section{COHORT DESCRIPTION}

A sample of ARDS survivors in German ICUs has been selected within the scope of the DACAPO study ('DACAPO: Surviving ARDS: the influence of quAlity of Care and individual Patient characteristics on quality Of life' funded by German Federal Ministry of Education and Research). The main objective of this study is to investigate the influence of QoC on HRQoL and RtW. With respect to the association between QoC and HRQoL/RtW, further hypotheses regarding moderating effects of sex, socioeconomic status, social support and psychopathological symptoms should be tested. A detailed protocol of the study has been published previously. ${ }^{13}$

By conducting additional follow-ups every 12 months after discharge from ICU, this initial sample will be transformed into the DACAPO (survivor) cohort. In particular, the DACAPO cohort will be set up to facilitate research in HCU and long-term psychiatric morbidity among ARDS survivors and to analyse long-term effects of QoC and HCU on HRQoL.

Between September 2014 and May 2016, 61 ICUs all over Germany included eligible patients with ARDS in the DACAPO ICU sample. Efforts were made to ensure that not only hospitals/ICUs specialised in the treatment of patients with ARDS (members of the ARDS Network Germany) participate, by reaching out to smaller urban or suburban hospitals. All participating clinics declared their willingness to include all eligible patients in the cohort during the period of recruitment. Written informed consent had to be provided by the patient. In patients who were cognitive incapable informed consent of patients' caregivers or legal guardians needed to be obtained.

Eligible patients had to meet following criteria for inclusion in the DACAPO ICU sample:

- ARDS is diagnosed in one of the participating ICUs or a referring hospital according to the 'Berlin definition'.

- Patient is older than 18 years at ARDS diagnosis.

In order to ensure maximal external validity of the DACAPO ICU sample, no further inclusion criteria and no exclusion criteria were applied.

The actual DACAPO cohort consists of the survivors of the DACAPO ICU sample (patients who were discharged alive from the ICU).

\section{Measurements}

During ICU treatment, a wide variety of sociodemographic, disease-related and care-related characteristics were recorded. Data acquisition was performed by means of web-based electronic case report forms (eCRFs). For this purpose, study nurses and physicians of every participating hospital were trained with regard to the detailed specification of the data that should be collected and the operation of the eCRFs.

Along with two short-term follow-up assessments (3 months and 6 months after ICU discharge), a yearly follow-up assessment is in progress.

Each follow-up consists of a paper-pencil questionnaire that is sent by post to the participant's home address. In order to minimise drop-out rates, we routinely mail a reminder letter and place a reminder phone call asking participants to complete and sent back the questionnaire. If there is no response to the reminder letters and reminder calls, we get in touch with the local resident registration office to receive information about whether the participant has died (mortality follow-up) or moved to another address.

\section{Exposure}

QoC is assessed in all participating ICUs. In cases where ARDS has been diagnosed in a referring hospital, QoC has been determined for the referring ICU as well. For this purpose, we apply the indicators of the quality assurance programme for intensive care implemented by the Deutsche Interdisziplinäre Vereinigung für Intensiv- und Notfallmedizin (German Interdisciplinary Association for Intensive and Emergency Medicine). ${ }^{14}$ These indicators are situated at the level of process and structural quality. In addition to this generic quality of intensive care indicator set, several ARDS specific variables have been incorporated. All indicators will be assessed by questionnaire at the institutional level.

If applicable, QoC was assessed on individual patient level for the interhospital transport between the referring hospitals and the participating ICUs. Particular attention was paid to indicators regarding process quality (medical equipment of the vehicle and medical qualifications of personnel). 


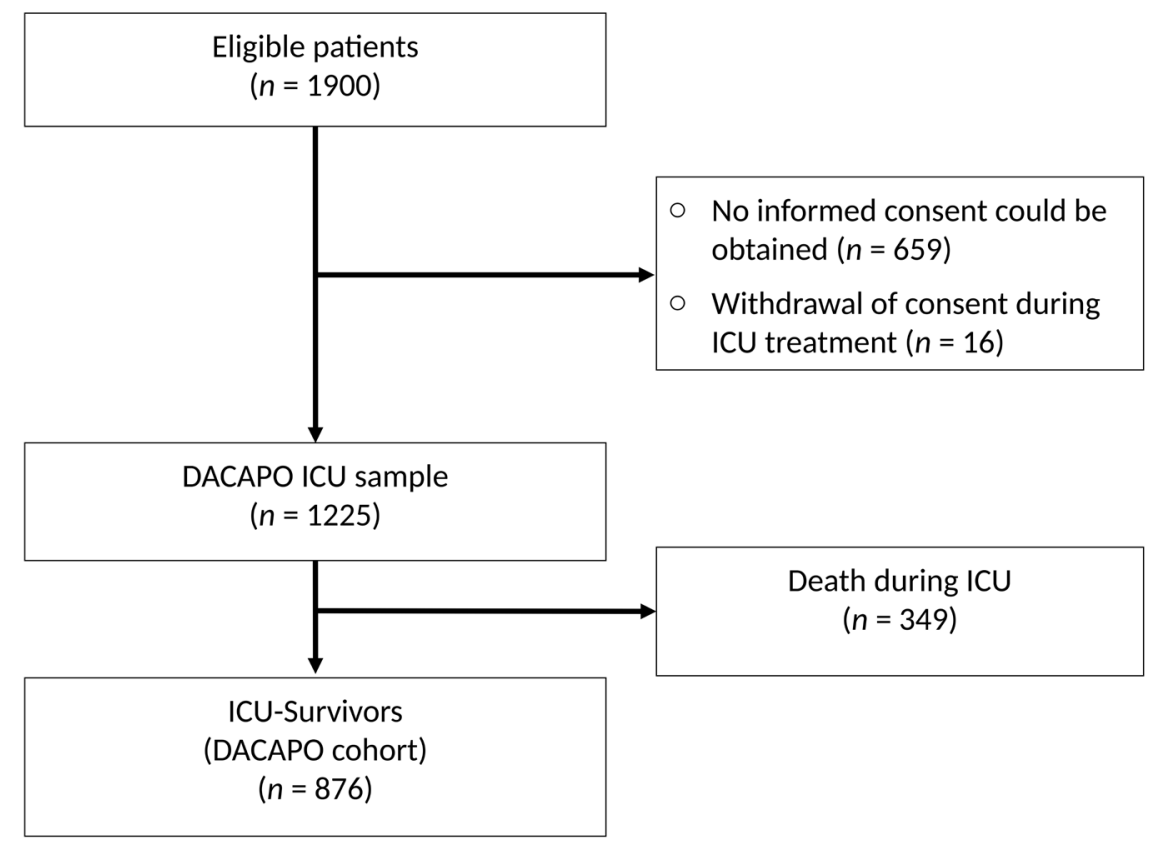

Figure 1 Patient flow of the DACAPO cohort. ICU, intensive care unit.

An important issue to be investigated based on the data of the DACAPO cohort is the long-term effects of HCU on HRQoL and RtW. Therefore, the follow-up questionnaires (starting from the 6-month follow-up) include questions regarding utilisation of ambulatory, inpatient and rehabilitative healthcare services.

\section{ICU baseline: moderator variables and covariates}

With regard to a valid assessment of morbidity and disease severity, several scores for morbidity and disease severity (Simplified Acute Physiology Score (SAPS) II, ${ }^{15}$ SAPS III, ${ }^{16}$ Sequential Organ Failure Assessment (SOFA) score ${ }^{17}$ have been recorded on item level for every patient at the time of (1) admission, (2) diagnosis of ARDS, (3) 24 hours after diagnosis and (4) discharge from ICU. For the sociodemographic characterisation age, gender, marital status, educational and professional level, living condition and socioeconomic status have been captured based on the information of the relatives/legal guardians. In addition, medical/ supportive treatment (ECMO, tracheotomy, nitric oxide (NO) inhalation, prone positioning and neuromuscular blockers) and adverse events (hypoglycaemia, hypoxia, accidental extubation and reintubation) have been assessed on individual patient level. In particular, most of the variables recorded in the phase of ICU treatment are intended to be covariates for the adjustment of disease severity, comorbidity and socioeconomic status in the final statistical model determining the influence of QoC on HRQoL and RtW. In addition, these medical baseline data (possibly combined with the exposure and/or outcome measurements) can serve as database for future clinical epidemiology research.

\section{Follow-up: outcomes}

The primary outcome is assessed by the Short Form-12 self-report questionnaire (SF-12), which has two scales (one for physical health and one for mental health). ${ }^{18}$

The secondary outcome RtW is determined by several items relating to date and extent of RtW.

\section{Follow-up: moderator variables and covariates}

Social support (emotional support, instrumental support and social integration) is assessed by using the F-SozU K-14. ${ }^{19}$ Symptoms of depression, panic disorder and alcohol abuse are assessed by the corresponding modules of the validated German version ${ }^{20} 21$ of the Patient Health Questionnaire (PHQ). ${ }^{22}$ With regard to the ICU stay, three categories of PTSD symptoms (emotional numbing, intrusion and hyperarousal) are determined by the screening tool Post-Traumatic Stress Syndrome 14-Questions Inventory (PTSS-14). ${ }^{23}$ Furthermore, the MacArthur Scale is used to determine subjective social status. This approach includes a rating of the perceived social position in the community on the scales of a ladder. ${ }^{24}$

\section{CHARACTERISTICS OF STUDY PARTICIPANTS}

Overall, 1900 patients with ARDS were enrolled in the electronic data capture system. Informed consent was obtained by 1225 of the patients or their legal guardians. The 876 ARDS survivors at the time of ICU discharge form the actual DACAPO cohort. A diagram of the patient flow is provided in figure 1. Descriptive statistics of sociodemographic and medical characteristics at the time of admission for the entire DACAPO ICU sample and for the DACAPO cohort (ICU survivors) are presented in table 1 . Small to moderate numbers of missing data in the medical variables are attributable to the 
Table 1 Selected baseline sociodemographic and medical characteristics of the DACAPO ICU sample and of the ICU survivors (initial DACAPO cohort)

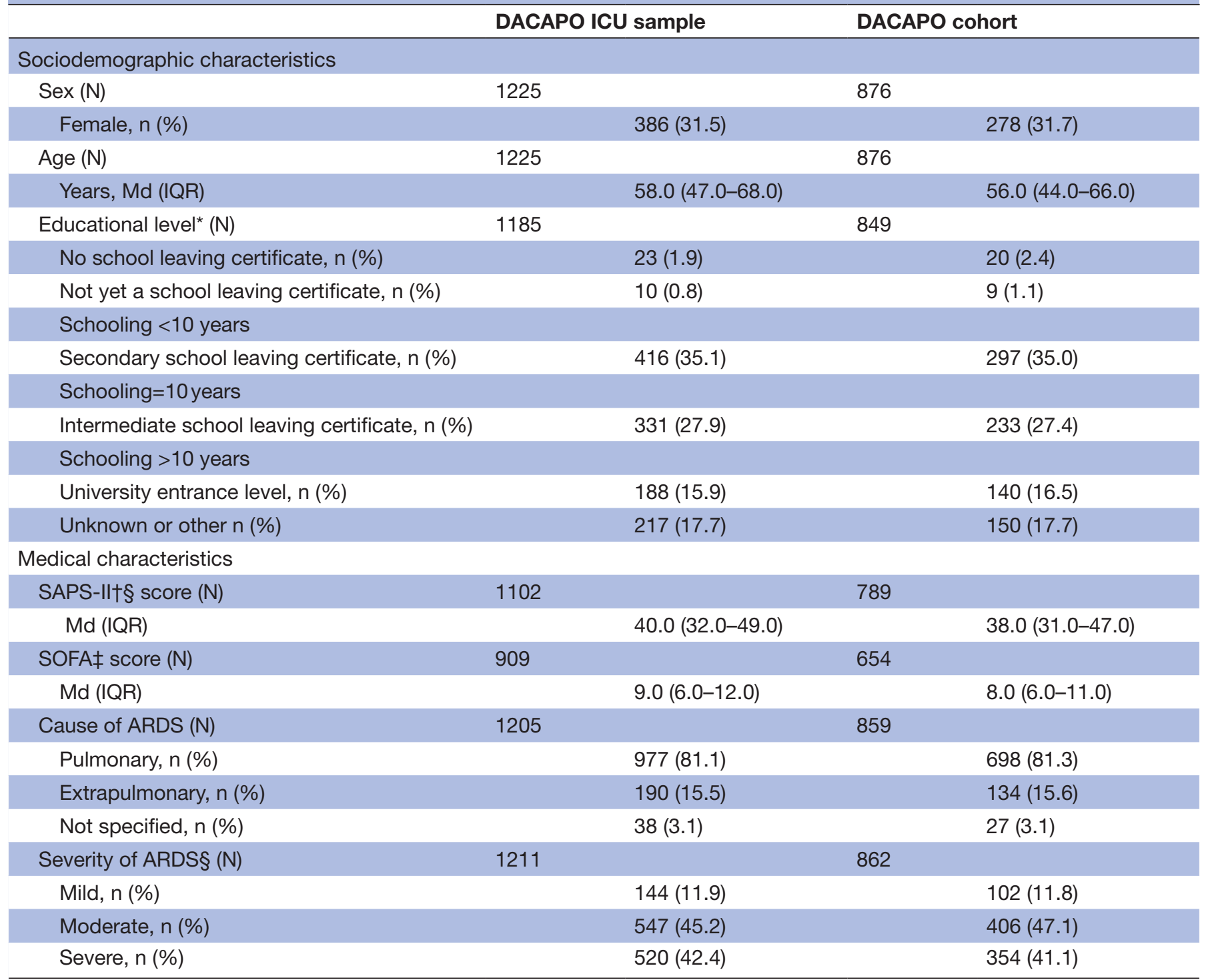

Note: numbers do not add up to $n=1225$ for all patients or to $n=876$ for survivors due to missing values.

'Data were provided by patients' caregivers/legal guardians.

$\dagger$ As assessed at admission at the DACAPO ICU.

†As assessed at time of ARDS diagnosis.

§SAPS-II score was calculated without the Glasgow Coma Scale.

ARDS, acute respiratory distress syndrome; ICU, intensive care unit; Md, median; SAPS, Simplified Acute Physiology Score; SOFA,

Sequential Organ Failure Assessment.

fact that many of the clinical and care-related characteristics are routinely recorded in the ICU setting.

\section{Findings to date}

The distribution of sex and age in our cohort is in perfect accordance with other large ARDS cohorts, which reveal that in particular older men are at higher risk for ARDS. Furthermore, the high prevalence of pulmonary diseases including pneumonia as most important risk factor of ARDS is in line with the scientific literature in this area. ${ }^{12}$

Taking a closer look at critical events (hypoxia, hypoglycaemia, unintended extubation and reintubation) and supportive measures (tracheotomy, NO inhalation,
ECMO, prone positioning and neuromuscular blockers) during ICU treatment, in particular the application of ECMO and prone positioning, were frequent, whereas critical events like hypoglycaemia also had a high prevalence. $^{12}$ These findings point out the potential for improvement in intensive care routines. However, the results reveal a comprehensive implementation of evidence-based measures like prone positioning ${ }^{25} 26$ and neuromuscular blockers. ${ }^{27}$

Against the background of changing diagnostic ARDS criteria, further comparisons of medical characteristics and outcomes between studies/cohorts should 


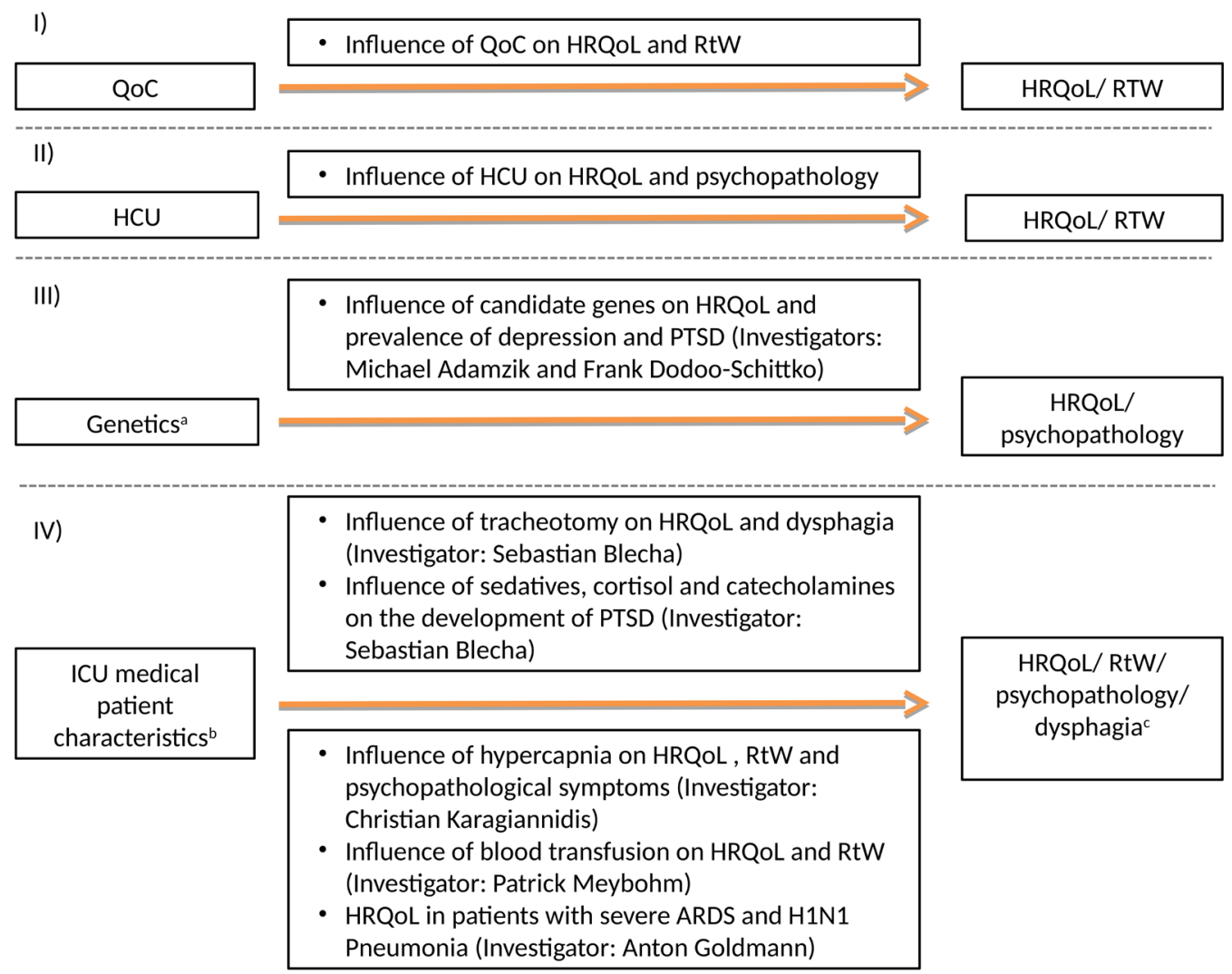

Figure 2 Main objectives and add-on projects using data of the DACAPO ICU sample/DACAPO cohort. (I) Main objective of the DACAPO study; (II) main objective of the DACAPO cohort; (III) genetic add-on project; and (IV) add-on projects with clinical background. ${ }^{a}$ An additional DNA analysis for the participants of the DACAPO cohort is intended. ${ }^{b}$ For some of the research questions, a retrospective collection of medical data is required. ${ }^{\circ}$ An additional follow-up with specific measurement instruments is intended for some research questions. ARDS, acute respiratory distress syndrome; HRQoL, health-related quality of life; HCU, healthcare utilisation; ICU, intensive care unit; PTSD, post-traumatic stress disorder; QoC, quality of care; RtW, return to work.

be drawn with caution. The Large observational study to UNderstand the Global impact of Severe Acute respirartory FailurE (LUNG SAFE) ${ }^{3}$ provides currently the only reliable sample of patients with ARDS corresponding to the current criteria of the 'Berlin definition'. The distribution of ARDS severity revealed by the LUNG SAFE sample differs from our findings. While LUNG SAFE reports the lowest proportion of persons with severe ARDS, the baseline data of our investigation indicate a much higher proportion of patients with severe ARDS and a lower proportion of patients with mild ARDS, but nevertheless the overall ICU mortality rate $(28.4 \%)$ is lower in our DACAPO ICU sample. ${ }^{312}$

\section{Strengths and limitations}

One of the strengths of the DACAPO cohort is the characterisation of ARDS survivors with a particular focus on sociodemographic conditions (education, socioeconomic status, marital status and so on). With regard to information bias, only validated instruments with satisfying psychometric properties are applied for psychometric constructs. A further strength is the broad spectrum of hospitals respectively ICUs that participated in patient recruitment. We were successful in involving university hospitals providing up-to-date apparatus and optimal personnel resources as well as smaller urban and suburban hospitals. This approach should reduce selection bias and ensure external validity. Additionally, in order to draw a representative sample/cohort of the general population of ARDS survivors, no exclusion criteria were applied.

However, the sample of the participating ICUs was drawn following a convenience sampling method, and the distribution of ARDS severity in the DACAPO ICU sample is not in accordance with the only large study ${ }^{3}$ applying the current Berlin definition of ARDS. ${ }^{2}$ The latter point tends to indicate that, although physicians of the participating ICUs have been trained in applying the diagnostic ARDS criteria of the Berlin definition, the mild form of ARDS is frequently overlooked in intensive care routine. This, in turn, would lead to a loss of representativeness of the DACAPO cohort and points out the need to screen all patients for eligibility in order to ensure representativeness, in particular if empirical and consensus-based and, therefore, non-salient syndromes like ARDS are investigated. Against this background, a strict implementation 
of an entity apart from the daily clinical routines that conducts the screening process every day would have been expedient. Because of the considerable number of participating ICUs, this approach was not feasible in practice.

Nevertheless, the DACAPO cohort is one of the largest cohorts of ARDS survivors described in literature and should be suitable for analytical issues related to associations between QoC, disease-related or care-related patient characteristics and physical, mental or social difficulties at the follow-up evaluations. At the same time, the DACAPO cohort enables taking into consideration important aspects of the individual sociodemographic conditions.

\section{Collaborations}

On the basis of a regulation given by the principal investigators of the study, every participating ICU that included at least one patient is encouraged to propose research questions that could be examined using the data provided by the DACAPO ICU sample, the DACAPO cohort and the data of the assessment of QoC. Figure 2 provides an overview of the main objective of the DACAPO study and envisaged projects going beyond.

The aim of a genetic add-on project is to investigate in ARDS survivors whether (1) there is an association between the NF-KB1 (-94 ins/del ATTG) promoter polymorphism and HRQoL and/or RtW and whether (2) the C957T polymorphism of dopamine D2 receptor gene and a single nucleotide polymorphism in SLC18A2 (rs363276) are associated with increased incidence of PTSD. Therefore, a DNA analysis of the DACAPO cohort participants using oral mucosa swabs is intended.

Furthermore, several add-on projects concerned with clinical topics of high relevance are currently under preparation. For some of these projects, the retrospective collection of additional medical ICU variables on individual patient level is required. For other research questions, an adapted follow-up questionnaire with additional measurement instruments (dysphagia, chronic pain and so on) has to be conducted (see figure 2). For details regarding the availability of data for potential new collaborators, see the data sharing section.

\section{Author affiliations}

${ }^{1}$ Medical Sociology, Institute of Epidemiology and Preventive Medicine, University of Regensburg, Regensburg, Germany

${ }^{2}$ Department of Anesthesia, Operative Intensive Care, University Hospital Regensburg, Regensburg, Germany

${ }^{3}$ Department of Anesthesiology, Emergency and Intensive Care Medicine, University Medicine, Georg-August-Universität Göttingen, Göttingen, Germany

${ }^{4}$ Department of Anesthesiology and Intensive Care Medicine, Campus VirchowKlinikum and Campus Charité Mitte, Charité-Universitätsmedizin Berlin, Berlin, Germany

${ }^{5}$ Department of Intensive Care Medicine, University Medical Centre Hamburg Eppendorf, Hamburg, Germany

${ }^{6}$ Department of Anesthesiology and Surgical Intensive Care, Medicine University Medical Center, Mannheim, Germany

${ }^{7}$ Department of Anesthesiology and Operative Intensive Care, University Hospital Bonn, Bonn, Germany

${ }^{8}$ Department of Anesthesiology and Intensive Care Medicine, University of Leipzig, Medical Faculty, Leipzig, Germany
${ }^{9}$ Department of Anesthesiology and Operative Intensive Care, University Hospital Münster, Münster, Germany

${ }^{10}$ Department of Anesthesiology and Operative Intensive Care, University Hospital

Marburg, Marburg, Germany

${ }^{11}$ Department of Anesthesiology, Intensive Care Medicine and Pain Therapy, University Hospital Frankfurt, Frankfurt, Germany

${ }^{12}$ Department of Anaesthesiology, University Hospital Knappschaftskrankenhaus Bochum, Bochum, Germany

${ }^{13}$ Department of Pneumology and Critical Care Medicine, Cologne-Merheim Hospital, ARDS and ECMO Centre, Cologne, Germany

Collaborators We are indebted to all the intensivists and study nurses throughout Germany who, with great commitment, recruited patients for the DACAPO study (the DACAPO Study Group): Aachen, Aachen University Hospital RWTH Aachen, Department of Anesthesiology (Dr Johannes Bickenbach, Dr Thorben Beeker, Dr Tobias Schürholz and Jessica Pezechk); Amberg, Klinikum Amberg, Department for Anaesthesiology (Dr Jens Schloer); Augsburg, Klinikum Augsburg (Dr Ulrich Jaschinski and Ilse Kummer); Bamberg, Sozialstiftung Bamberg Hospital, Department for Anaesthesiology (Dr Oliver Kuckein); Berlin, Charité - University Medicine Berlin, Department of Anaesthesiology and Intensive Care Medicine (Dr Steffen Weber-Carstens, Dr Anton Goldmann, Dr Stefan Angermair and Krista Stoycheva); Berlin, HELIOS Klinikum Berlin-Buch, Department of Intensive Care Medicine (Professor Dr Jörg Brederlau, Nadja Rieckehr, Gabriele Schreiber and Henriette Haennicke); Bielefeld, Ev. Krankenhaus Bielefeld, Department of Anesthesiology, Intensive Care Medicine, Emergency Medicine and Pain Therapy (Dr Friedhelm Bach, Dr Immo Gummelt, Dr Silke Haas, Catharina Middeke, Dr Ina Vedder and Marion Klaproth); Bochum, Ruhr University Bochum, Department of Anaesthesiology (Professor Dr Michael Adamzik, Dr Jan Karlik, Dr Stefan Martini and Luisa Robitzky); Bonn, University Hospital Bonn, Department of Anesthesiology and Intensive Care Medicine (Professor Dr Christian Putensen, Dr Thomas Muders and Ute Lohmer); Bremen, Klinikum Bremen-Mitte, Department of Anesthesiology (Professor Dr Rolf Dembinski); Deggendorf, Medical Center, Department of Anaesthesiology and Intensive Care Medicine (Dr Petra Schäffner and Dr Petra Wulff-Werner); Dortmund, Klinikum Dortmund, Department of Critical Care Medicine (Elke Landsiedel-Mechenbier, Daniela Nickoleit-Bitzenberger and Ann-Kathrin Silber); Dresden, University Hospital Dresden Carl Gustav Carus, Department of Anesthesiology and Intensive Care Medicine (Professor Dr Maximilian Ragaller, Professor Dr Marcello Gama de Abreu, Alin Ulbricht and Linda Reisbach); Frankfurt am Main, University Hospital Frankfurt, Department of Anaethesiology, Intensive Care Medicine and Pain Therapy (Professor Dr Kai Zacharowski, Professor Dr Patrick Meybohm, Gerhard Schwarzmann, Simone Lindau, Carolin Wiedenbeck and Johannes Reske); Freiburg, University Medical Center Freiburg, Department of Anaesthesiology and Critical Care Medicine Freiburg (Professor Dr Alexander Hötzel and Dr Johannes Kalbhenn); Freising, Klinikum Freising, Department of Anaesthesiology (Dr Christoph Metz and Dr Stefan Haschka); Göppingen, Klinik am Eichert, ALB FILS Kliniken, Department of Anaesthesiology and Intensive Care (Dr Stefan Rauch); Göttingen, University Medical Center, Department of Anaesthesiology, Emergency and Intensive Care Medicine (Professor Dr Michael Quintel, Dr Lars-Olav Harnisch, Dr Sophie Baumann and Andrea Kernchen); Greifswald, University Medicine Greifswald, Department of Internal Medicine B (Dr Sigrun Friesecke and Sebastian Maletzki); Hamburg, University Hospital Hamburg-Eppendorf, Department of Intensive Care Medicine, Center of Anesthesiology and Intensive Care Medicine (Professor Dr Stefan Kluge, Dr Olaf Boenisch, Dr Daniel Frings, Birgit Füllekrug, Dr Nils Jahn, Dr Knut Kampe, Grit Ringeis, Brigitte Singer and Dr Robin Wüstenberg); Hannover, Hannover Medical School, Department of Anaesthesiology and Intensive Care Medicine (Dr Jörg Ahrens, Dr Heiner Ruschulte, Dr Andre Gerdes and Dr Matthias Groß); Hannover, Hannover Medical School, Department of Respiratory Medicine (Dr Olaf Wiesner and Aleksandra Bayat-Graw); Heidelberg, University of Heidelberg, Department of Anaesthesiology (Dr Thorsten Brenner, Dr Felix Schmitt and Anna Lipinski); Herford, Klinikum Herford, Clinic for Anaesthesiology, Surgical Intensive Care Medicine, Emergency Care Medicine, Pain Management (Professor Dr Dietrich Henzler, Dr Klaas Eickmeyer, Dr Juliane Krebs and Iris Rodenberg); Homburg, Homburg University Medical Centre, Department of Anaesthesiology, Intensive Care and Pain Medicine (Dr Heinrich Groesdonk, Kathrin Meiers, Karen Salm and Professor Dr Thomas Volk); Ibbenbüren, Ibbenbüren General Hospital, Division of Thoracic Surgery and Lung Support (Professor Dr Stefan Fischer and Basam Redwan); Immenstadt, Kempten-Oberallgaeu Hospitals, Clinic for Pneumology, Thoracic Oncology, Sleep- and Respiratory Critical Care (Dr Martin Schmölz, Dr Kathrin Schumann-Stoiber and Simone Eberl); Ingolstadt, Klinikum Ingolstadt, Department of Anaesthesiology and Critical Care Medicine (Professor Dr Gunther Lenz, Thomas von Wernitz-Keibel and Monika Zackel); Jena, Jena 
University Hospital, Department of Anesthesiology and Intensive Care Therapy (Dr Frank Bloos, Dr Petra Bloos, Anke Braune, Anja Haucke, Almut Noack, Steffi Kolanos, Heike Kuhnsch and Karina Knuhr-Kohlberg); Kassel, Klinikum Kassel, Department of Anaesthesiology (Dr Markus Gehling); Kempten, Klinikum Kempten-Oberallgäu gGmbH, Department for Anesthesia and Operative Intensive Care (Professor Dr Mathias Haller, Dr Anne Sturm and Dr Jannik Rossenbach); Kiel, University Medical Center Schleswig-Holstein, Campus Kiel, Department of Anesthesiology and Intensive Care Medicine (Dr Dirk Schädler and Stefanie D'Aria); Köln, CologneMerheim Hospital, Department of Pneumology and Critical Care Medicine (Professor Dr Christian Karagiannidis, Dr Stephan Straßmann, Professor Dr Wolfram Windisch); Köln, University Hospital of Cologne, Department of Anaesthesiology and Intensive Care Medicine (Professor Dr Thorsten Annecke and Dr Holger Herff); Langen, Asklepios Kliniken Langen-Seligenstadt, Department of Anesthesiology and Intensive Care Medicine (Dr Michael Schütz); Leipzig, University of Leipzig, Department of Anesthesiology and Intensive Care Medicine (Dr Sven Bercker, Hannah Reising, Mandy Dathe and Christian Schlegel); Ludwigsburg, Klinikum Ludwigsburg, Academic Teaching Hospital, University of Heidelberg, Department of Anaesthesiology (Katrin Lichy); Ludwigshafen, Klinikum Ludwigshafen, Department of Anesthesiology and Intensive Care Medicine (Professor Dr Wolfgang Zink and Dr Jana Kötteritzsch); Mainz, University Medical Center Mainz, Department of Anaesthesiology (Dr Marc Bodenstein, Susanne Mauff and Peter Straub); Magdeburg, Magdeburg University Medical Centre, Department of Anaesthesiology and Intensive Care Medicine (Dr Christof Strang, Florian Prätsch and Professor Dr Thomas Hachenberg); Mannheim, University Medical Center Mannheim, Department of Anaesthesiology and Surgical Intensive Care Medicine (Dr Thomas Kirschning, Dr Thomas Friedrich and Dr Dennis Mangold); Marburg, University Hospital, Department of Anaesthesiology (Dr Christian Arndt and Tilo Koch); Mönchengladbach, Kliniken Maria-Hilf GmbH, Department of Cardiology (Dr Hendrik Haake and Katrin Offermanns); München, Bogenhausen Hospital, Department of Anaesthesiology (Professor Dr Patrick Friederich and Dr Florian Bingold); München, Klinikum Großhadern, Department of Anaesthesiology (Dr Michael Irlbeck, Professor Dr Bernhard Zwissler); München, Klinikum Neuperlach, Städtisches Klinikum München GmbH, Department of Anesthesiology, Critical Care and Pain Medicine (Dr Ines Kaufmann); München, Klinikum rechts der Isar, Department for Anaesthesiology of the Technical University of Munich (Dr Ralph Bogdanski, Dr Barbara Kapfer, Dr Markus Heim and Dr Günther Edenharter); Münster, University Hospital Münster, Department for Anaesthesiology, Intensive Care Medicine and Pain Therapy (Professor Dr Björn Ellger and Daniela Bause); Neumarkt, Kliniken des Landkreises Neumarkt i.d.OPf, Department for Anaesthesiology and Intensive Care Medicine (Dr Götz Gerresheim); Nürnberg, General Hospital Nuremberg, Paracelsus Medical University, Department of Emergency Medicine and Intensive Care (Dr Dorothea Muschner, Professor Dr Michael Christ and Arnim Geise); Osnabrück, Marienhospital Osnabrück, Department of Anaesthesiology (Dr Martin Beiderlinden and Dr Thorsten Heuter); Passau, Klinikum Passau, Department for Anaesthesiology (Dr Alexander Wipfel); Regensburg, Caritas Krankenhaus St. Josef, Department for Anaesthesiology (Dr Werner Kargl, Dr Marion Harth and Dr Christian Englmeier); Regensburg, Regensburg University Hospital, Department of Anaesthesiology, Operative Intensive Care (Professor Dr Thomas Bein, Dr Sebastian Blecha, Dr Kathrin Thomann-Hackner and Marius Zeder); Stuttgart, Katharinenhospital, Department of Anesthesiology (Dr Markus Stephan); Traunstein, Klinikum Traunstein, Department of Anaesthesiology (Dr Martin Glaser); Tübingen, Tübingen University Hospital, Eberhard-Karls University Tübingen, Department of Anaesthesiology and Intensive Care Medicine (Dr Helene Häberle); UIm, Ulm University, Department of Anesthesiology (Professor Dr Hendrik Bracht, Christian Heer and Theresa Mast); Würzburg, University of Würzburg, Department of Anaesthesia and Critical Care (Dr Markus Kredel and Dr Ralf Müllenbach).

Contributors FD-S analysed, interpreted the data and wrote the manuscript; SuB helped writing the manuscript and also reviewed it for important intellectual content; MB, SeB, MQ, SW-C, SK, TK, TM, SvB, BE, ChristianAr, PM, MA, AG and CK reviewed the manuscript for important intellectual content; TB contributed his knowledge and expertise in the field of intensive care and reviewed the manuscript for important intellectual content; ChristianAp helped in writing the manuscript and reviewed it for important intellectual content. All authors have read and approved the final version of the manuscript.

Funding The study is funded by the German Ministry of Education and Research (Bundesministerium für Bildung und Forschung, funding number 01GY1340).

Competing interests None declared.

Patient consent Obtained.

Ethics approval Ethics Committee of the University of Regensburg.
Provenance and peer review Not commissioned; externally peer reviewed.

Data sharing statement Data are available depending on permission from the original data holders (the two principal investigators (PIs) Christian Apfelbacher and Thomas Bein). Potential new collaborators should send a brief proposal including a sketch indicating the envisaged analysis project to the PIs. Due to data economy and subsequently data protection, only the variables required for the analysis project will be provided.

Open Access This is an Open Access article distributed in accordance with the Creative Commons Attribution Non Commercial (CC BY-NC 4.0) license, which permits others to distribute, remix, adapt, build upon this work non-commercially, and license their derivative works on different terms, provided the original work is properly cited and the use is non-commercial. See: http://creativecommons.org/ licenses/by-nc/4.0/

(c) Article author(s) (or their employer(s) unless otherwise stated in the text of the article) 2018. All rights reserved. No commercial use is permitted unless otherwise expressly granted.

\section{REFERENCES}

1. Pierrakos $\mathrm{C}$, Karanikolas M, Scolletta S, et al. Acute respiratory distress syndrome: pathophysiology and therapeutic options. J Clin Med Res 2012;4:7-16.

2. Ranieri VM, Rubenfeld GD, Thompson BT, et al. Acute respiratory distress syndrome: the Berlin Definition. JAMA 2012;307:2526-33.

3. Bellani G, Laffey JG, Pham T, et al. Epidemiology, patterns of care, and mortality for patients with acute respiratory distress syndrome in intensive care units in 50 Countries. JAMA 2016;315:788-800.

4. Scholten EL, Beitler JR, Prisk GK, et al. Treatment of acute respiratory distress syndrome with prone positioning. Chest 2016.

5. Sen A, Callisen HE, Alwardt CM, et al. Adult venovenous extracorporeal membrane oxygenation for severe respiratory failure: Current status and future perspectives. Ann Card Anaesth 2016;19:97-111.

6. Dowdy DW, Eid MP, Dennison CR, et al. Quality of life after acute respiratory distress syndrome: a meta-analysis. Intensive Care Med 2006;32:1115-24.

7. McHorney CA, Ware JE, Raczek AE. The MOS 36-Item short-form health survey (SF-36): II. Psychometric and clinical tests of validity in measuring physical and mental health constructs. Med Care 1993;31:247-63.

8. Herridge MS, Tansey CM, Matté A, et al. Functional disability 5 years after acute respiratory distress syndrome. N Engl J Med 2011;364:1293-304.

9. Bienvenu OJ, Colantuoni E, Mendez-Tellez PA, et al. Cooccurrence of and remission from general anxiety, depression, and posttraumatic stress disorder symptoms after acute lung injury: a 2-year longitudinal study. Crit Care Med 2015;43:642-53.

10. Davydow DS, Desai SV, Needham DM, et al. Psychiatric morbidity in survivors of the acute respiratory distress syndrome: a systematic review. Psychosom Med 2008;70:512-9.

11. Dodoo-Schittko F, Brandstetter S, Blecha S, et al. Determinants of quality of life and return to work following acute respiratory distress syndrome. Dtsch Arztebl Int 2017:114:103-9.

12. Dodoo-Schittko F, Brandstetter S, Brandl M, et al. Characteristics and provision of care of patients with the acute respiratory distress syndrome: descriptive findings from the DACAPO cohort baseline and comparison with international findings. J Thorac Dis 2017:9:818-30.

13. Brandstetter S, Dodoo-Schittko F, Blecha S, et al. Influence of quality of care and individual patient characteristics on quality of life and return to work in survivors of the acute respiratory distress syndrome: protocol for a prospective, observational, multi-centre patient cohort study (DACAPO). BMC Health Serv Res 2015;15:563.

14. Braun JP, Kumpf O, Deja M, et al. The German quality indicators in intensive care medicine 2013--second edition. Ger Med Sci 2013;11.

15. Le Gall JR, Lemeshow S, Saulnier F. A new simplified acute physiology score (saps ii) based on a european/north american multicenter study. JAMA 1993;270:2957-63.

16. Moreno RP, Metnitz PG, Almeida E, et al. SAPS 3--From evaluation of the patient to evaluation of the intensive care unit. Part 2: Development of a prognostic model for hospital mortality at ICU admission. Intensive Care Med 2005;31:1345-55.

17. Vincent JL, Moreno R, Takala J, et al. The SOFA (Sepsis-related Organ Failure Assessment) score to describe organ dysfunction/ failure. On behalf of the Working Group on Sepsis-Related Problems 
of the European Society of Intensive Care Medicine. Intensive Care Med 1996;22:707-10.

18. Ware J, Kosinski M, Keller SD. A 12-Item short-form health survey: construction of scales and preliminary tests of reliability and validity. Med Care 1996;34:220-33.

19. Fydrich T, Sommer G, Brähler E. Social support questionnaire (F-SozU) manual. Göttingen, Germany: Hogrefe, 2007.

20. Löwe B, Spitzer RL, Zipfel S, et al. PHQ-D Health questionnaire for patients. Manual]. Pfizer GmbH, Karlsruhe 2002.

21. Gräfe KZS, Herzog W, Löwe B. Screening for psychiatric disorders with the Patient Health Questionnaire (PHQ). Results from the German validation study]. Diagnostica 2004;50:11.

22. Spitzer RL, Williams JB, Kroenke $\mathrm{K}$, et al. Validity and utility of the PRIME-MD patient health questionnaire in assessment of 3000 obstetric-gynecologic patients: the PRIME-MD Patient Health Questionnaire Obstetrics-Gynecology Study. Am J Obstet Gynecol 2000;183:759-69.
23. Radtke FM, Franck M, Drews T, et al. The post-traumatic stress syndrome 14-Questions Inventory (PTSS-14) - Translation of the UK-PTSS-14 and validation of the German version. Anasthesiol Intensivmed Notfallmed Schmerzther 2010;45:688-95.

24. Miyakawa M, Magnusson Hanson LL, Theorell T, et al. Subjective social status: its determinants and association with health in the Swedish working population (the SLOSH study). Eur J Public Health 2012;22:593-7

25. Park SY, Kim HJ, Yoo KH, et al. The efficacy and safety of prone positioning in adults patients with acute respiratory distress syndrome: a meta-analysis of randomized controlled trials. J Thorac Dis 2015;7:356-67.

26. Guérin C, Reignier J, Richard JC, et al. Prone positioning in severe acute respiratory distress syndrome. N Engl J Med 2013;368:2159-68.

27. Papazian L, Forel JM, Gacouin A, et al. Neuromuscular blockers in early acute respiratory distress syndrome. $N$ Engl J Med 2010;363:1107-16. 